\title{
Discovery of Discrete Structured Bubbles within Lunar Regolith Impact Glasses
}

\author{
Marek S. Żbik, ${ }^{1}$ Yen-Fang Song, ${ }^{2}$ Chun-Chieh Wang, ${ }^{2}$ and Ray L. Frost ${ }^{1}$ \\ ${ }^{1}$ Discipline of Chemistry, Faculty of Science and Technology, Queensland University of Technology, 2 George Street, \\ GPO Box 2434, Brisbane, Qld 4001, Australia \\ ${ }^{2}$ National Synchrotron Radiation Research Center, 101 Hsin-Ann Road, Hsinchu Science Park, Hsinchu 30076, Taiwan
}

Correspondence should be addressed to Ray L. Frost, r.frost@qut.edu.au

Received 20 December 2011; Accepted 10 January 2012

Academic Editors: S. Ettori and R. A. West

Copyright ( 2012 Marek S. Żbik et al. This is an open access article distributed under the Creative Commons Attribution License, which permits unrestricted use, distribution, and reproduction in any medium, provided the original work is properly cited.

\begin{abstract}
The unusual morphology and internal structure of bubbles within lunar regolith impact glasses have been studied using traditional scanning electron microscopy and the novel technique transmission X-ray microscopy (TXM), with 3D tomography reconstruction. Here, we show the previously unknown phenomenon of building a highly porous cellular structure within bubbles in glassy particles of the dust fraction of lunar regolith. Vesicles within studied lunar glasses are filled in with submicron-sized particles as shown in the presented micrograph. These particles consist of glass nano in size elements. What is shown in the TXM tomography reconstruction anaglyph demonstrates cellular-like, 3D structure where oblique probably glassy fine particles down to $100 \mathrm{~nm}$ in diameter build chains of sophisticated network. It also may be suggested that submicron and nano-sized grains present in lunar regolith are the result of particle liberation from broken glassy vesicles. This liberation takes place when regolith is exposed to constant impact pulverisation. Liberated particles are permanently enriching lunar soil in the finest soil constituent. This constituent presence in lunar regolith may be responsible for the unusual behaviour of lunar material. This unusual constituent of lunar regolith and its properties have to be better understood before our permanent lunar exploration begins.
\end{abstract}

Suggestions that the lunar surface be covered by a layer of loose regolith were based on the impact model of its genesis. The lunar surface is covered by numerous impact craters whose diameters vary from tens of kilometers to being microscopic in size. Continuous pulverisation of lunar surface rocks and constant mixing develops matured type of soil which is unknown on Earth.

The first accurate estimation of lunar soil grain size was published by Wesselink [1] where he shows that the grains of the lunar powder must be smaller than $0.3 \mathrm{~mm}$. Subsequent measurements on the lunar regolith samples delivered from the moon during Apollo and Luna missions confirmed Wesselink's estimations and added accurate date from direct measurements $[2,3]$. Only $10 \mathrm{wt} \%$ of the lunar regolith grains was found to be larger than $0.25 \mathrm{~mm}$, and most grains were below $10 \mu \mathrm{m}$ in size. Żbik et al. [4] described the presence of mostly submicron-sized particles building the lunar fine dust aggregates and suggesting their impact origin.
The lunar regolith sample studied came from the depth of $15-18 \mathrm{~cm}$ below the lunar surface and was drilled and delivered on Earth by the first ever automatic lunar mission "Luna 16." This sample has been obtained from the Academy of Sciences USSR [5]. The impact features within this sample were described [6]. Lunar soil, called regolith, consists of larger rock fragments, mineral grains, breccias, and vesicular glass which often weld other soil particles together. Most of the lunar regolith glassy particles and breccia grains are porous and display voids in the form of vesicular bubbles which vary in size as shown in SEM micrographs, Figure 1. Vesicles within studied lunar glasses are not smooth and are filled in with submicron-sized particles as shown in SEM micrograph Figure 2. These particles form the finest lunar dust features, and consist of glass microdroplets and their broken irregular fragments. These lunar regolith constituents were noticed before but never discussed, and their presence was mostly overlooked and treated as the soil contaminants. 


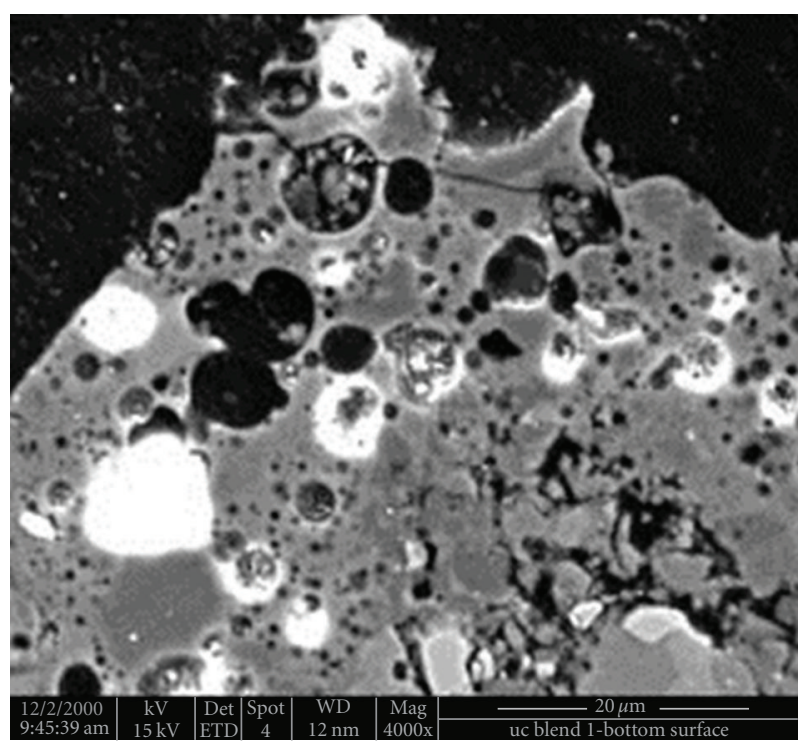

Figure 1: SEM micrographs of studied lunar soil morphology show glassy fragment larger breccia grain of $800 \mu \mathrm{m}$ in diameter decorated by fine vesicular voids (bubbles).

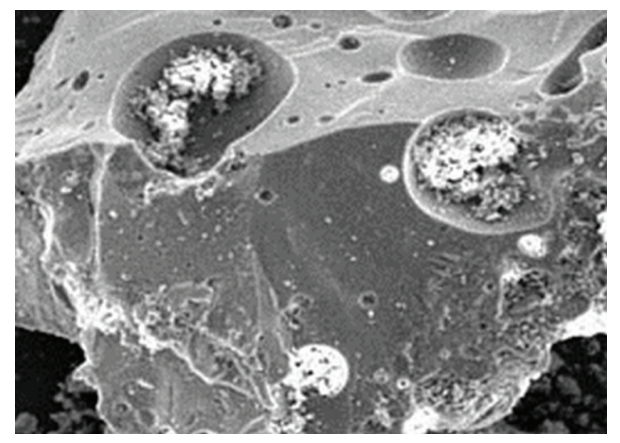

FIGURE 2: SEM micrographs of studied lunar soil morphology show that fine, submicron dust particles fill the broken bubbles'interior; frame width is $10 \mu \mathrm{m}$.

Recently, a revolutionary new technique has been implemented to the study of nanomaterial science, called nanotomography [7]. The transmission X-ray microscope (TXM) with $60 \mathrm{~nm}$ tomographic resolution has been installed at beamline BL01B of NSRRC in Taiwan. This microscope works with a synchrotron photon source [8]. This new technique [9] has recently been used to investigate soil samples without sample prepreparation. This is the big advantage of TXM tomographic study over other microscopy methods.

In the typical TXM, 3D reconstruction image has been shown in Figure 3. In this micrograph, the fine structure of submicron-sized particles arrangement within bubble has been revealed. As shown in Figure 3, the TXM tomography reconstruction anaglyph demonstrates cellular 3D structure where oblique probably glassy fine particles down to $100 \mathrm{~nm}$ in diameter build chains of sophisticated network. The space arrangement of these nanoparticles looks to span all the

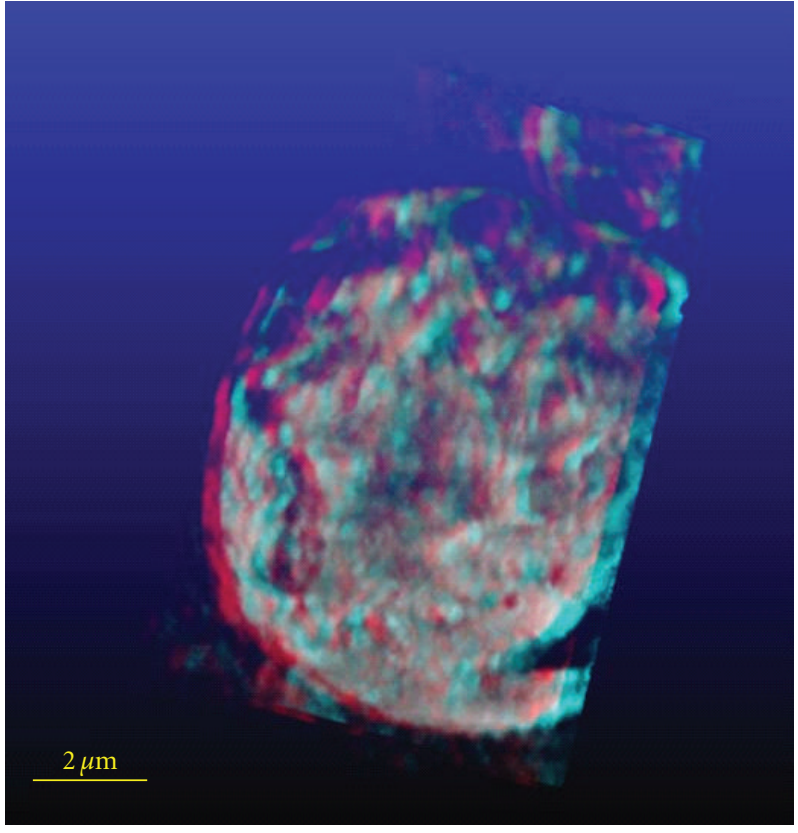

FIGURE 3: TXM tomography reconstruction anaglyph shows highly porous cellular structure encapsulated within vesicular void (bubble) in glassy fragment of the lunar breccias; frame width is $10 \mu \mathrm{m}$.

volume of bubble and is shaped in the form of cellular structure with large up to $500 \mathrm{~nm}$ voids. Other few bubbles studied show similar values. To apply certain concepts to quantify with innerbubble aggregate structure, it requires larger volume scans, and the study will be continued on other lunar glassy samples where larger volume of material can be studied.

Described structural and morphological features of the lunar regolith are novel and have never been studied or seen before. Walls and bubbles internal space is discreetly structured and it is perhaps the effect of impact processes in low-viscosity melt. In earthen impact glass and tektites [10], the bubble walls are smooth, and the interior is empty. Structures like revealed within the lunar impact glass bubbles have never been described, and it may be specific to the lunar material only.

It also may be suggested that submicron and nano-sized grains present in lunar regolith are the result of particle liberation from the broken glassy vesicles. This liberation takes place when regolith is exposed to constant impact pulverisation. Liberated particles are permanently enriching lunar soil in finest soil constituent. This constituent presence in lunar regolith may be responsible for the unusual behaviour [11] of lunar material like stickiness, britches, and electrostatic hovering. In our view, presented result of $\mathrm{X}$ ray inner bubble morphology led us to a very important discovery which improves our knowledge about the origin and evolution of lunar topsoil. This unusual constituent of lunar regolith and its properties has to be better understood before our permanent lunar exploration begins. 


\section{References}

[1] A. J. Wesselink, "Heat conductivity and nature of lunar surface material," Bulletin of the Astronomical Institutes of the Netherlands, vol. 10, pp. 351-363, 1948.

[2] J. C. Laul, J. J. Papike, and S. B. Simon, "The lunar regolith: comparative studies of the Apollo and Luna 16, 20 and 24," in Proceedings of the 12th Lunar and Planetary Science Conference, pp. 389-407, 1981.

[3] I. I. Czerkasow and W. W. Schwarew, Gruntoviedienie Luny, Nauka, Moscow, 1979.

[4] M. S. Żbik, R. L. Frost, Y.-F. Song, and Y.-M. Chen, "High porosity chained aggregates from the topsoil of the Lunar regolith Dust," in Proceedings of the 9th Australian Space Conference, pp. 55-65, Australian Academy of Science, University of Sydney, 2010.

[5] B. Grabowska-Olszewska and M. Żbik, "Lunar Soil Sample in Poland," Przeglad Geologiczny, no. 7, pp. 418-420, 1984.

[6] M. Żbik, "Features of impact metamorphism in lunar regolith studies," Bulletin of the Polish Academy of Sciences, Earth Sciences, vol. 39, pp. 299-309, 1991.

[7] D. Attwood, "Microscopy: nanotomography comes of age," Nature, vol. 442, no. 7103, pp. 642-643, 2006.

[8] G. C. Yin, M. T. Tang, Y. F. Song et al., "Energy-tunable transmission $\mathrm{X}$-ray microscope for differential contrast imaging with near $60 \mathrm{~nm}$ resolution tomography," Applied Physics Letters, vol. 88, no. 24, Article ID 241115, 2006.

[9] M. S. Żbik, R. L. Frost, and Y. F. Song, "Advantages and limitations of the synchrotron based transmission X-ray microscopy in the study of the clay aggregate structure in aqueous suspensions," Journal of Colloid and Interface Science, vol. 319, no. 1, pp. 169-174, 2008.

[10] M. Żbik, M. Jasieniak, and R. S. C. Smart, "Organosilane occurrence in irghizite samples from the Zhamanshin impact crater, Kazakhstan," Meteoritics and Planetary Science, vol. 35, no. 5, pp. 943-947, 2000.

[11] B. O'Brien, "Direct active measurements of movements of lunar dust: rocket exhausts and natural effects contaminating and cleansing Apollo hardware on the Moon in 1969," Geophysical Research Letters, vol. 36, no. 9, Article ID L09201, 2009. 

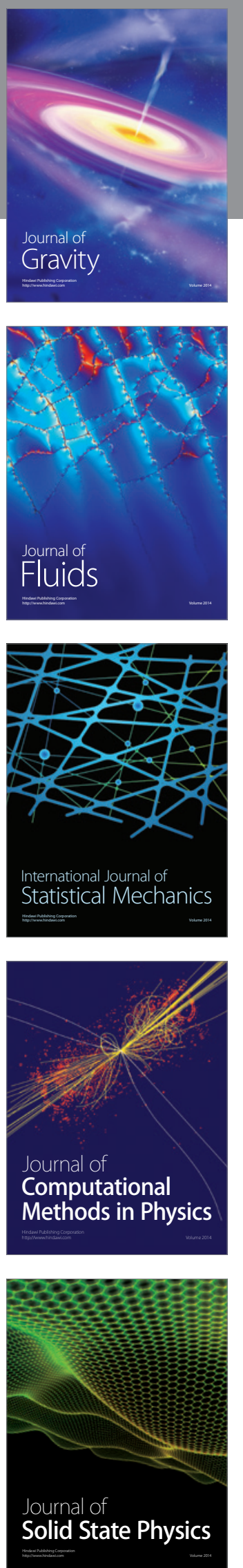

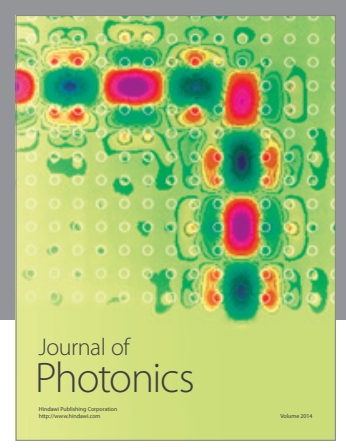

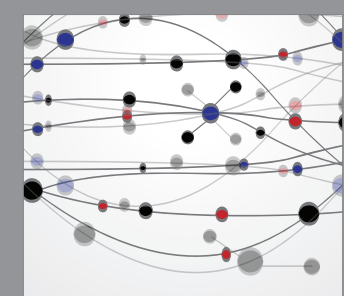

The Scientific World Journal
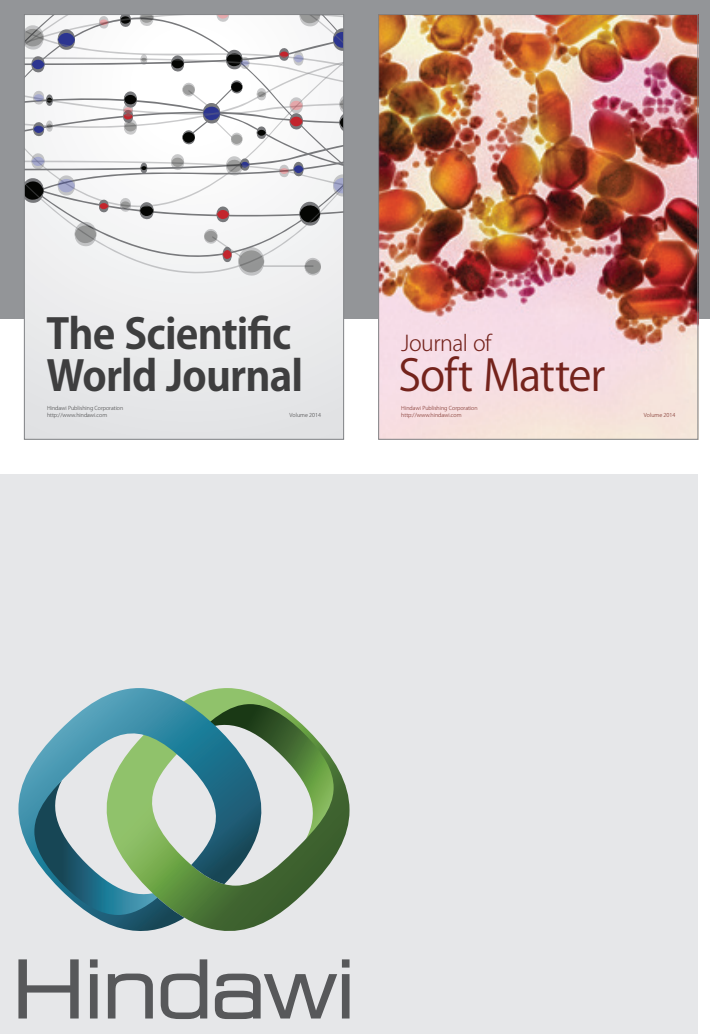

Submit your manuscripts at

http://www.hindawi.com
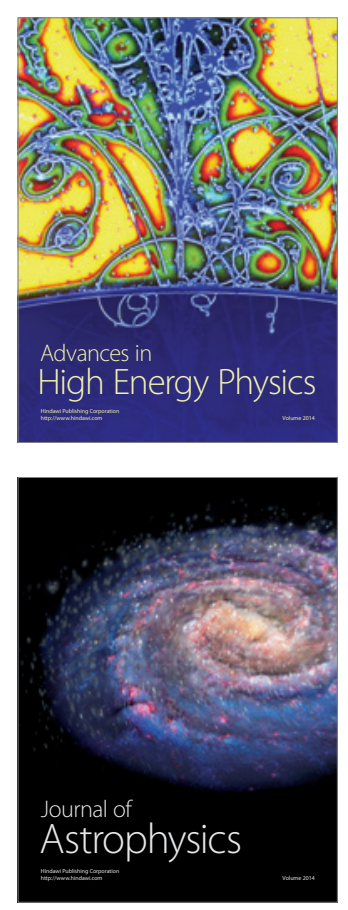
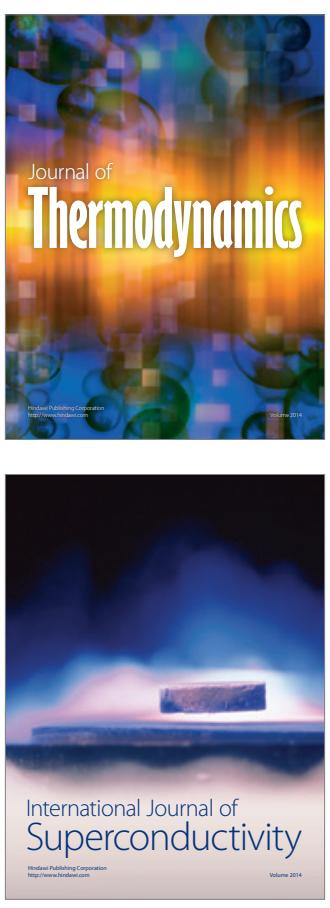
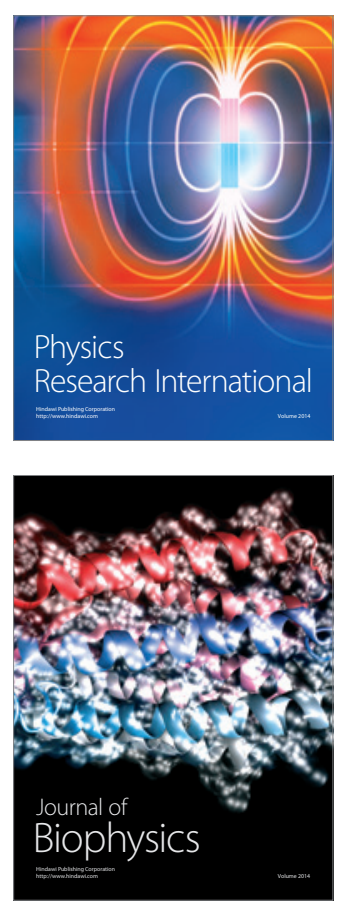
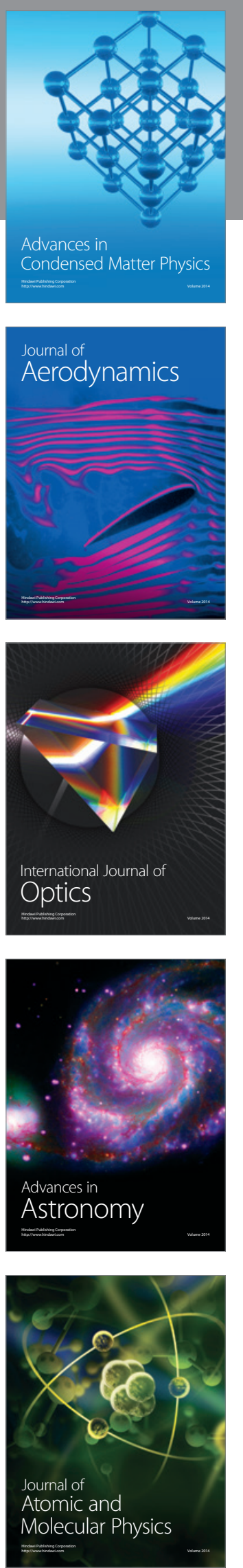